\title{
Adverse interaction effects of chronic and acute sleep deficits on spatial working memory but not on verbal working memory or declarative memory
}

\author{
Eva Hennecke $^{1}$ (D) | Denise Lange ${ }^{1,2}$ | Florian Steenbergen ${ }^{1}$ | Judith Fronczek-Poncelet ${ }^{3}$ | \\ David Elmenhorst $^{3,4}$ | Andreas Bauer ${ }^{3,5}$ | Daniel Aeschbach ${ }^{1,6,7}$ | \\ Eva-Maria Elmenhorst ${ }^{1,2}$ (D)
}

\author{
${ }^{1}$ Department of Sleep and Human Factors \\ Research, Institute of Aerospace Medicine, \\ German Aerospace Center, Cologne, \\ Germany \\ ${ }^{2}$ Institute for Occupational and Social \\ Medicine, Medical Faculty, RWTH Aachen \\ University, Aachen, Germany \\ ${ }^{3}$ Institute of Neuroscience and Medicine \\ (INM-2), Forschungszentrum Jülich, Jülich, \\ Germany \\ ${ }^{4}$ Department of Psychiatry and \\ Psychotherapy, Rheinische Friedrich- \\ Wilhelms-University Bonn, Bonn, Germany \\ ${ }^{5}$ Neurological Department, Medical Faculty, \\ Heinrich-Heine-University, Düsseldorf, \\ Germany \\ ${ }^{6}$ Division of Sleep and Circadian Disorders, \\ Brigham and Women's Hospital, Boston, \\ MA, USA \\ ${ }^{7}$ Division of Sleep Medicine, Harvard \\ Medical School, Boston, MA, USA
}

\section{Correspondence}

Eva-Maria Elmenhorst, Institute of Aerospace Medicine, German Aerospace Center (DLR), Linder Höhe, 51147 Köln, Germany.

Email: eva-maria.elmenhorst@dlr.de

Funding information

The Authors would like to gratefully acknowledge the financial support and endorsement from the DLR Management Board Young Research Group Leader Program and the Executive Board Member for Space Research and Technology.

\begin{abstract}
The accumulation of chronic sleep deficits combined with acute sleep loss is common in shift workers and increases the risk of errors and accidents. We investigated single and combined effects of chronic and acute sleep loss and recovery sleep on working memory performance ( $N$-back task) and on overnight declarative memory recall (paired-associate lists) in 36 healthy participants. After baseline measurements, the chronic sleep restriction group ( $n=21$; mean [SD] age 26 [4] years) underwent 5 nights of sleep restriction (5-hr time in bed [TIB]), whereas the control group ( $n=15$; mean [SD] age 28 [6] years) had 8-hr TIB during those nights. Afterwards, both groups spent 1 night with 8-hr TIB prior to acute sleep deprivation for $38 \mathrm{hr}$, and a final recovery night (10-hr TIB). Chronic sleep restriction decreased spatial N-back performance compared to baseline (omissions: $p=.001$; sensitivity: $p=.012$ ), but not letter $\mathrm{N}$-back performance or word-pair recall. Acute sleep deprivation impaired spatial $\mathrm{N}$-back performance more in the chronic sleep restriction group than in the control group (interaction between group and time awake: $p \leq .02$ ). No group differences during acute sleep loss appeared in letter $N$-back performance or word recall. It is concluded that chronic sleep loss, even when followed by a night of recovery sleep, increases the vulnerability to impairments in spatial working memory during subsequent acute sleep loss. Verbal working memory and declarative memory were not affected by restricted sleep.
\end{abstract}

\section{KEYWORDS}

cognition, polysomnography, sleep deprivation

\section{1 | INTRODUCTION}

Sleep of sufficient duration is crucial for cognitive performance. For adults a habitual sleep duration of $\geq 7 \mathrm{hr}$ has been recommended to maintain health (Hirshkowitz et al., 2015; Watson et al., 2015). 
chronically restricted sleep and acute sleep deprivation (Dinges et al., 1997; Elmenhorst et al., 2018; Van Dongen et al., 2003), there is some debate about the degree of performance impairments in executive functions like working memory (Chee \& Choo, 2004 Dinges et al., 1997; Gohar et al., 2009; Groeger et al., 2008; Jiang et al., 2011; Lo et al., 2012; Tucker et al., 2010; Van Dongen et al., 2003). As attentional processes are impaired by sleep deprivation but supposed to underlie higher-order cognitive functions according to the vigilance hypothesis (Lim \& Dinges, 2010), findings of minor working memory impairments only after sleep deprivation might be surprising. However, according to the controlled attention hypothesis (Lim \& Dinges, 2010; Pilcher et al., 2007), especially monotonous tasks require top-down control, which is assumed to be compromised by sleep deficits. Thus, the engaging aspect of executive tasks may counteract attentional deficits due to sleep loss.

According to the multicomponent model (Baddeley \& Hitch, 1974), working memory consists of a central executive, a phonological loop, and a visuospatial sketchpad. Accordingly, previous studies showed differences in brain areas involved in verbal and spatial working memory tasks (Smith et al., 1996). Working memory is an essential component of many cognitive processes enabling execution of complex tasks e.g. mental arithmetic (Baddeley, 2010; Baddeley \& Hitch, 1974). Lo et al. (2012) compared the effects of 7 nights of sleep restriction (6-hr time in bed [TIB]) and additional acute sleep loss on different cognitive domains in adults. Their results suggest that sustained attention, as measured by a psychomotor vigilance task, was much more affected by chronic sleep restriction than working memory performance in an $\mathrm{N}$-back task.

Working memory function is tightly related to the functioning of long-term memory (Hartshorne \& Makovski, 2019). It has been suggested that sleep after learning enhances declarative memory consolidation, whereas wakefulness shows no beneficial effects on memory formation (Gais et al., 2006; Plihal \& Born, 1997; Tucker et al., 2006). Within sleep, the prevalence of electroencephalography (EEG) slow waves $(\sim 0.5-4.5 \mathrm{~Hz})$ was found to benefit various types of learning (Aeschbach et al., 2008) and declarative memory consolidation in particular (Backhaus et al., 2007; Diekelmann \& Born, 2010; Gais \& Born, 2004; Rasch \& Born, 2013; Tucker et al., 2006).

Slow-wave sleep (SWS) during the first hours after sleep onset typically reflects sleep pressure after a period of wakefulness (Aeschbach \& Borbely, 1993; Borbely, 1982; Borbely et al., 1981). When sleep is restricted, sleep architecture adapts, favouring SWS at the expense of rapid eye movement sleep (REM) (Belenky et al., 2003; Elmenhorst et al., 2008). Due to these homeostatic processes, a large proportion of SWS is preserved during shortened sleep. Consistent with these findings shortened sleep showed no effect on declarative memory consolidation and recall in several previous studies (Cedernaes et al., 2016; Kopasz et al., 2010; Lo et al., 2016; Plihal \& Born, 1997; Tucker \& Fishbein, 2009; Voderholzer et al., 2011).
Although previous studies suggested that working memory and declarative memory consolidation are less affected by sleep restriction, an important aspect that has not been assessed is whether a history of sleep restriction is associated with impaired memory performance during acute sleep deprivation. Furthermore, the possible moderating role of a single night of recovery sleep, both after chronic and acute sleep loss, should be assessed.

The aim of the present study was to examine the single and combined effects of chronic and acute sleep deprivation and recovery sleep on working memory performance in an $\mathrm{N}$-back task (verbal and spatial) and on overnight declarative memory recall of paired-associate lists. On the basis of previous studies, we hypothesised adverse effects of chronic sleep restriction on working memory, but not on declarative memory recall. We expected that a recovery night of 8-hr TIB would not be sufficient to completely recover impairments in working memory performance due to sleep restriction. Instead, residual effects of chronic sleep loss may become manifest during subsequent extended wakefulness (Cohen et al., 2010). Accordingly, we hypothesised that impairments in working memory performance during total sleep deprivation (TSD) would be larger with a prior history of sleep restriction compared to absence of a chronic sleep deficit. In contrast, given that the effect of sleep restriction on SWS was anticipated to be small, we did not expect to find an effect of chronic sleep loss on declarative memory recall.

\section{2 | METHODS}

\section{1 | Participants}

Volunteers underwent a selection procedure involving questionnaires (general health status, substance abuse, sleep habits/abnormalities, psychological screening) and a physical examination including blood and urine testing. Only healthy, non-smoking women and men with regular sleep times were included. A total of 36 individuals, aged 20-39 years, participated and were randomly assigned to either a control (15 individuals, mean [SD] age 28 [6] years; five females) or a chronic sleep restriction group (21 individuals, mean [SD] age 26 [4] years; nine females). The study was approved by the Ethics Committee of the North Rhine Medical Board. Participants gave written informed consent prior to participation.

\section{2 | Procedure}

At 1 week prior to the laboratory stay, participants adhered to a sleep-wake protocol with TIB from 22:00-07:00 or 23:0008:00 hours to ensure sleep satiation. Adherence was checked with actigraphs and sleep diaries. Participants were instructed to abstain from alcohol and caffeine the week before and during the stay in the laboratory. Participants were under constant supervision and were allowed to do non-vigorous activities only. Lights were dimmed to $<100$ lux at the angle of gaze during wake times and to $<3$ lux during scheduled sleep times, and the temperature was kept constant. 
Control group

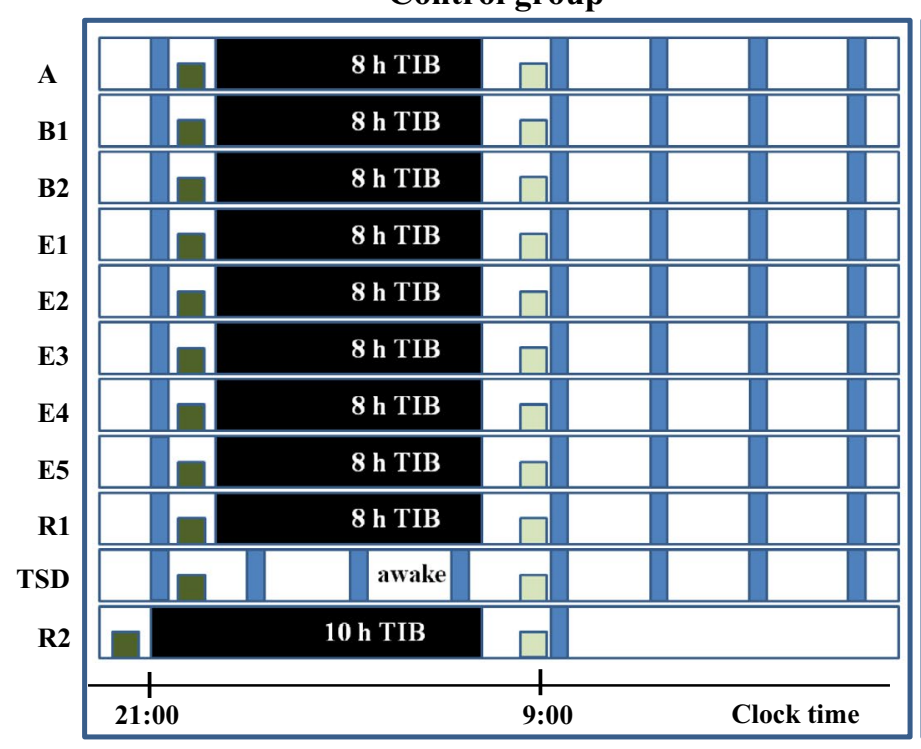

Chronic sleep restriction group

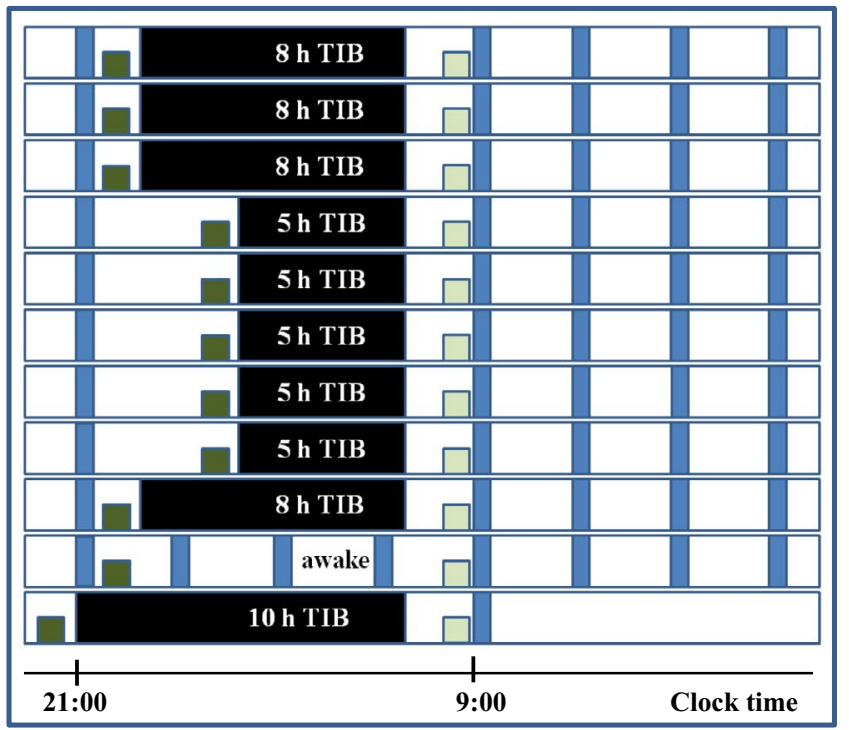

Time in bed (TIB), Polysomnography

N-Back task

Learning phase of the declarative memory task

Retrieval phase of the declarative memory task

FIGURE 1 Study design of the control and the chronic sleep restriction group. Days are displayed as rows: A, adaptation; B1, 2, baseline 1, 2; E1-E5, experimental days 1-5 (chronic sleep restriction group: chronic sleep restriction; control group: normal sleep); R1, 2, recovery 1, 2; TSD, total sleep deprivation; TIB, time in bed

Upon arrival, urine tests were performed to exclude substance use and pregnancy. Figure 1 gives an overview of the study design.

Participants spent 1 adaptation night and 2 baseline nights with 8-hr TIB (23:00-07:00 or 24:00-08:00 hours) in the laboratory before being exposed to either normal 8-hr TIB (control group) or to chronic sleep restriction to 5-hr TIB (02:00-07:00 or 03:00-08:00 hours, chronic sleep restriction group) for 5 nights. After that, both groups had an 8 -hr recovery night (23:00-07:00 or 24:00-08:00 hours) followed by $38 \mathrm{hr}$ of acute TSD and a 10-hr recovery night (21:00-07:00 or 22:00-08:00 hours).

Every evening, $1 \mathrm{hr}$ before bedtime (TSD: after $15 \mathrm{hr}$ of wakefulness), the learning phase of the paired-associate lists was scheduled. The recall phase in the morning was scheduled $1.75 \mathrm{hr}$ after getting up (TSD: after $25.75 \mathrm{hr}$ of wakefulness). At 3-hourly intervals during wakefulness, starting with the word recall, participants performed a 35-min cognitive test battery. It was performed in quiet, single rooms without time cues.

\section{3 | Materials}

\subsection{1 | N-back task}

Working memory capacity was assessed with a letter and a spatial version of the N-back task (Gevins \& Cutillo, 1993). Participants were asked to indicate whether the currently displayed stimulus matched that of $N$ steps earlier regarding the letter and position, respectively. Every session started with the letter N-back. At first, a "1-back" trial block was performed, after a short break 2- and 3-back trial blocks followed. Thereafter, the spatial N-back with 1-, 2-, and 3-back trials followed. For more information on the $\mathrm{N}$-back task see Supporting Information.

\subsection{2 | Paired-associate lists}

An adapted version (addition of new word pairs and randomisation according to our study requirements) of the paired-associate lists task (Plihal \& Born, 1997) was used to test overnight declarative memory performance. For results of a previously conducted study with the same lists of word pairs please see Basner et al. (2011). In the evening 32 new, semantically related word pairs (German nouns, e.g. vacation - tent) were successively presented and had to be recalled in a cued recall procedure immediately ( $\geq 60 \%$ of the words had to be correctly recalled, otherwise the learning phase was repeated) and the next morning. The first and last four word pairs were excluded from analysis to prevent primacy and recency effects. For detailed information see Supporting Information.

\subsection{3 | Polysomnography}

Electrodes were attached according to the international 10-20 system (EEG: F4-A1, C4-A1, O2-A1, F3-A2, C3-A2, O1-A2; 
electrocardiography [ECG]; and electromyography [EMG]). During the first night, additional sensors were attached to check for the absence of sleep-related disorders. One participant had an increased apneahypopnea index $(\mathrm{AHI}=16.3)$, which was detected after participation. As this participant did neither show associated cortical arousals during sleep nor increased sleepiness, we did not remove the datasets from the sample. Sampling rate was $256 \mathrm{~Hz}$. The EEG signal was amplified with a time constant of $2.3 \mathrm{~s}$ and low-pass filtered $(-6 \mathrm{~dB}$ at $70 \mathrm{~Hz})$.

\section{4 | Data reduction and analyses}

Data were analysed with SAS University Edition (2019) and SPSS (version 21). Normal distribution was checked. The significance level of 0.05 was adjusted for multiple testing according to BonferroniHolm. All statistical tests were two-sided. Effect sizes omega ${ }^{2}\left(\omega^{2}\right)$ and partial eta ${ }^{2}\left(\eta_{\mathrm{p}}{ }^{2}\right)$ for mixed models were calculated according to Tippey and Longnecker (2016). For $\omega^{2}$ and $\eta_{p}{ }^{2}$ values of .01, .06 and .14 have been suggested to represent small, medium and large effects, respectively (Ellis, 2010; Kirk, 1996). For post hoc comparisons, effect size was calculated with the correlation coefficient $r$.

\subsection{1 $\mid \mathrm{N}$-back task}

Correct responses (hits), false alarms and omissions were calculated. Sensitivity $\left(A^{\prime}\right)$ was obtained and analysed (Stanislaw \& Todorov, 1999).

The morning difference between baseline day 2 (B2) and key conditions (experimental day 5 [E5], recovery day 1 [R1], TSD, and recovery day 2 [R2]) were calculated. All analyses include 1-, 2- and 3-back values for each participant and condition/time point. The morning measures were analysed as we obtained a morning value only for R2. Moreover, morning measures were obtained at the same circadian time regarding the declarative memory task. Mixed analyses of variance (ANOVAs; SAS proc mixed) for repeated measures (random factor: "subject"; fixed factors: "group", "condition" [E5, R1, TSD, R2], and "group $\times$ condition") and post hoc pairwise comparisons provided by the SAS least-square means (Ismeans) statement $t$-test compared conditions between groups, aiming at detecting group differences at key experimental days at the same circadian time points. Effect sizes were calculated for the interactions and key post hoc comparisons.

Separate ANOVAs per group were performed to reduce effects of individual variability of performance in general and especially of performance impairments in response to sleep loss. Effects between baseline and key study days were assessed within groups using the random factor "subject" and the fixed factors "condition" (B2, E5, R1, TSD, R2), "load" and their interaction (post hoc: Ismeans t-test). Load describes the varying cognitive load in 1-, 2-, and 3-back trials.

Impairments in omissions and sensitivity (expressed as difference to baseline) were analysed with mixed ANOVAs (random factor: "subject"; fixed factors: "group", "time awake" and their interaction) for tests performed in the 38-hr period between the end of the first recovery night until the start of the second recovery night to investigate the differences between groups in the time course of performance impairments during additional acute wakefulness. Effect sizes were calculated for the group $\times$ time awake interactions. Time course of performance within groups was analysed (absolute values), using mixed ANOVAs (random factor: "subject"; fixed factor: "time awake").

\subsection{2 | Paired-associate lists}

Data sets of two chronic sleep restriction group participants had to be excluded because tests were not performed at the scheduled time.

Encoding: The number of learning trials and the number of correctly recalled words in the last learning trial were calculated and compared between groups with Mann-Whitney U-tests (exact) and within groups with Wilcoxon signed-rank tests.

Delayed recall: The difference between correctly recalled words in the evening and in the matutinal retrieval phase was calculated.

A mixed model for repeated measures was used to compare performance across groups and conditions (E5, R1, TSD, R2). The effect size for the group $\times$ condition interaction was calculated. Mixed models were calculated for each group separately comparing key experimental days (E5, R1, TSD, R2) to B2 (post hoc: Ismeans t-test).

\subsection{3 | Polysomnography}

Sleep period time (SPT), sleep onset latency (SOL, first occurrence of N2 or deeper sleep), N3 latency, minutes spent in N1, N2, N3, REM and Wake (American Academy of Sleep Medicine [AASM] criteria (Iber et al., 2007)) were compared across groups (Mann-Whitney Utests) and within groups (Wilcoxon signed-rank tests). Minutes spent in N3 in E5 in the sleep restriction group were correlated with morning performance on $\mathrm{E} 5$.

\section{3 | RESULTS}

\section{1 | Working memory}

Figure 2 gives an overview of the $\mathrm{N}$-back morning performance of the two groups. ANOVAs showed no interaction between group and condition (E5, R1, TSD, R2) concerning the letter N-back, but a main effect of group and condition concerning omissions (group $\times$ condition: $F(3$, $390)=2.51, p=.06, \omega^{2}=.007, \eta_{p}{ }^{2}=.017$; group: $F(1,34)=5.79, p=$ .02 ; condition: $F(3,390)=66.03, p<.001$ ) as well as sensitivity (group $x$ condition: $F(3,389)=1.58, p=.19, \omega^{2}=.003, \eta_{p}{ }^{2}=.01$; group: $F(1$, $33.5)=5.83, p=.02$; condition: $F(3,389)=61.9, p<.001)$. In contrast, significant interactions appeared regarding the spatial $\mathrm{N}$-back in omissions (group $\times$ condition: $F(3,390)=4.06, p=.007, \omega^{2}=.01, \eta_{p}{ }^{2}=.028$; group: $F(1,34)=.68, p=.42$; condition: $F(3,390)=81.93, p<.0001)$ and sensitivity (group $\times$ condition: $F(3,389)=3.94, p=.009, \omega^{2}=.01$, 
FIGURE 2 Comparison of $N$-back morning performance under different conditions between control and chronic sleep restriction group. The number of (a) omissions and (b) sensitivity on the $\mathrm{N}$-back task expressed as difference to baseline day 2 is displayed separately for control (white) and chronic sleep restriction (grey) group and for letter (left column) and spatial (right column) $\mathrm{N}$-back. The mean is indicated by the " $\mathrm{x}$ ". $\mathrm{E} 5$, experimental night 5; R1, 2, recovery 1, 2; TSD, total sleep deprivation. Please note that there are no significant differences regarding post hoc pairwise comparisons between groups and conditions

\section{Letter}

(a)

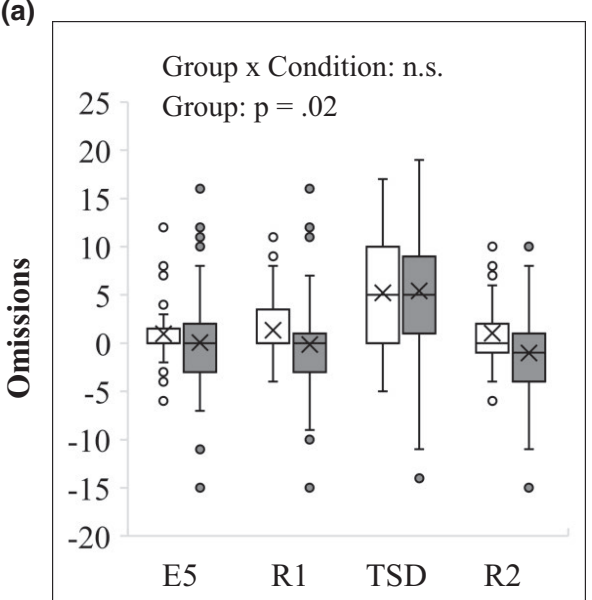

(b)

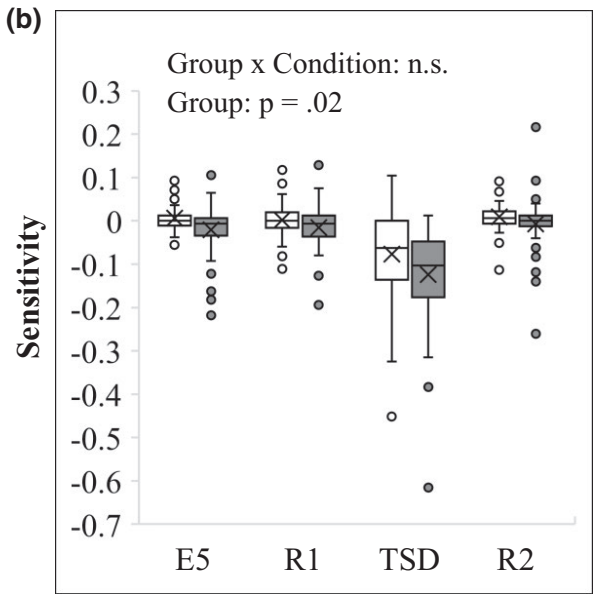

Condition
Spatial
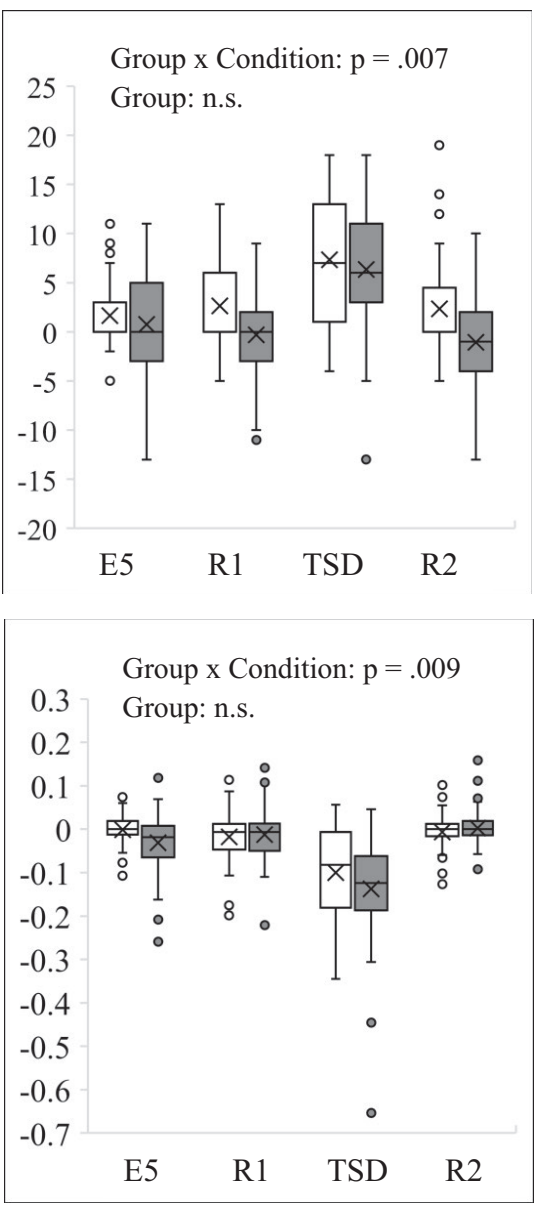

Condition $\eta_{\mathrm{p}}{ }^{2}=.027$; group: $F(1,34)=.81, p=.38$; condition: $F(3,389)=78.97$, $p<.0001)$. Pairwise comparisons indicated no group differences during the key conditions after correction for multiple testing, which was supported by low effect sizes for spatial $N$-back $(r \leq .23)$ and letter $N$ back $(r \leq .28)$ except for the letter omissions $N$-back group comparison at TSD with a medium effect size $(r=.32)$. However, the group difference at TSD on spatial $\mathrm{N}$-back sensitivity just missed significance after correction for multiple testing (alpha Bonferroni-Holm Step 1: .05/5 = $.01 ; p=.0384, r=.23$ ). For information on confidence intervals (Cls) see Table S1, the course of $N$-back performance over all conditions (B2, E1-E5, R1, TSD, R2) is shown in Figure S1.

Within-group analyses showed that in both groups morning performance was significantly influenced by condition (B2, E5, R1, TSD, R2) and load of the $\mathrm{N}$-back tasks without an interaction (Table S2). Pairwise comparisons indicated impaired performance during acute sleep deprivation as compared to baseline for all variables (letter and spatial $\mathrm{N}$ back omissions and sensitivity) in the control and in the chronic sleep restriction group (all $p<.0001$ ). In addition, performance was impaired after sleep restriction compared to baseline in the spatial $\mathrm{N}$-back (omissions: $p=.001$; sensitivity: $p=.012$ ), but not the letter $N$-back.
In a next step, mixed ANOVAs were calculated to assess the effect of prior sleep restriction on the time course of $\mathrm{N}$-back performance during TSD. Concerning the letter $\mathrm{N}$-back, there was no interaction between group and time awake for omissions (group $\times$ time awake: $F(11,1,238)=1.38, p=.17, \omega^{2}=.003, \eta_{p}{ }^{2}$ $=.012$; group: $F(1,34)=4.13, p=.05$; time awake: $F(11,1,238)=$ $31.92, p<.0001$ ) and sensitivity (group $\times$ time awake: $F(11,1,232)$ $=1.28, p=.23, \omega^{2}=.002, \eta_{p}{ }^{2}=.011$; group $(1,33.6)=4.72, p=$ .04 ; time awake: $F(11,1,232)=25.43, p<.0001)$. A significant interaction between group and time awake was found for the spatial $N$-back, reflecting more omissions (group $\times$ time awake: $F(11,1,238$ ) $=2.07, p=.02, \omega^{2}=.007, \eta_{p}^{2}=.017$; group: $F(1,34)=.71, p=.41$; time awake: $F(11,1,238)=49.55, p<.0001)$ and lower sensitivity (group $\times$ time awake: $F(11,1,234)=2.29, p=.009, \omega^{2}=.008, \eta_{p}{ }^{2}$ $=.019$; group: $F(1,34)=.57, p=.45$; time awake: $F(11,1,234)=$ $42.26, p<.0001)$ following sleep restriction. Figure 3 shows performance during acute sleep deprivation including information on within-group comparisons (Ismeans t-test) between the tests performed during acute sleep deprivation and the test performed $2 \mathrm{hr}$ after the preceding 8-hr recovery night. 


\section{2 | Declarative memory}

Encoding: The number of learning trials (group mean) needed to reach criterion (i.e. correct recall rate of $\geq 60 \%$ ) was between 1 and 1.28 for both groups and all conditions. The number of correctly recalled words in the last learning trial (immediate recall) was between 19.5 and 21.5. There were no between- or within-group differences in the number of learning trials or correctly recalled words in the last learning trial of each evening session (all $p>.05$ ). For more information see Table S3.

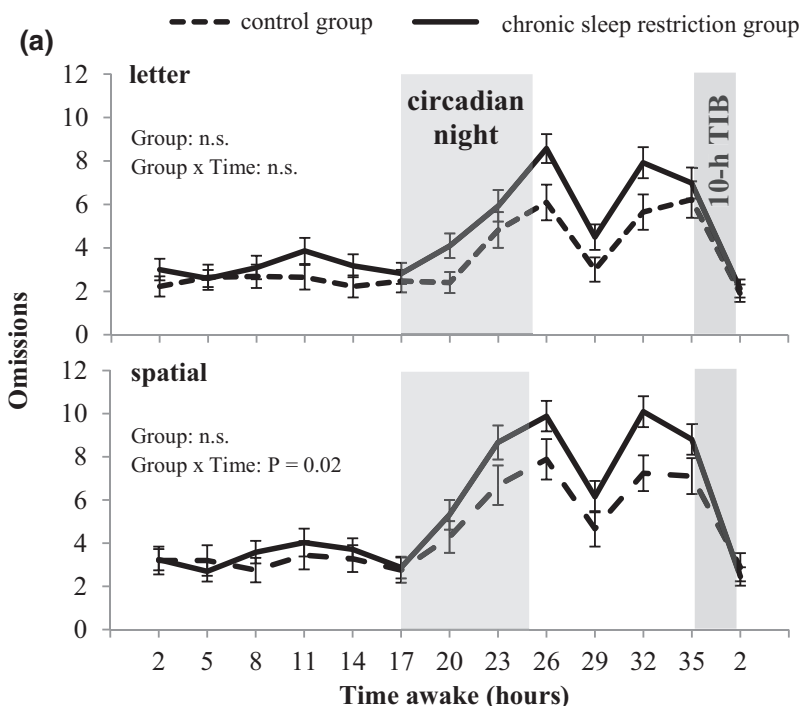

(b)

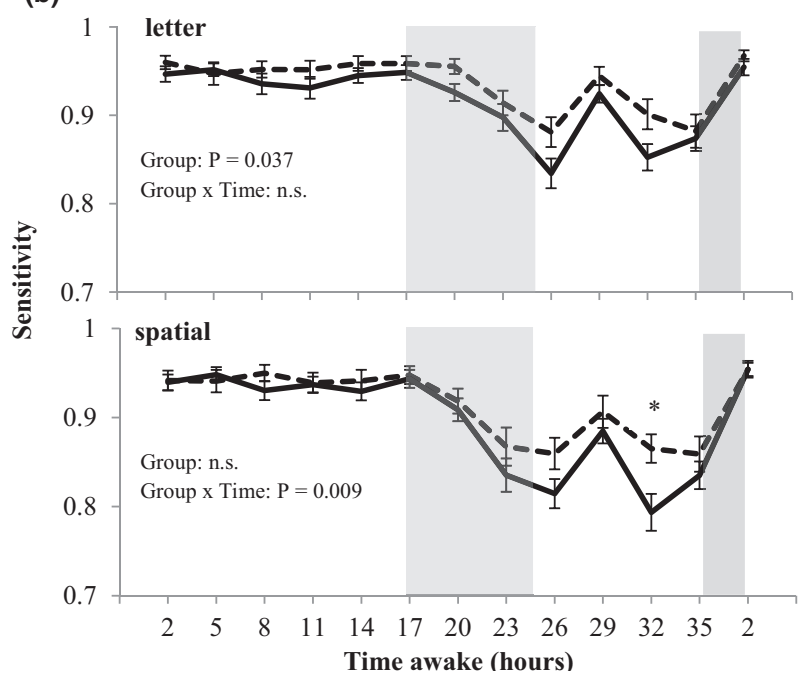

FIGURE $3 \quad$ N-Back performance of the two groups during acute sleep deprivation. The number of (a) omissions (raw value, group means \pm standard errors) and (b) sensitivity of the $\mathrm{N}$-back task is shown from the first $2 \mathrm{hr}$ awake after the 8-hr recovery night to $35 \mathrm{hr}$ of wakefulness and $2 \mathrm{hr}$ after awakening from the 10-hr recovery night. Significant differences between groups are indicated $(*)$. Within both groups, significant differences to baseline $(p<.002)$ appeared after 23, 26, 32 and $35 \mathrm{hr}$ of wakefulness. In the chronic sleep restriction group, spatial $\mathrm{N}$-back performance additionally differed from baseline after $29 \mathrm{hr}$ of wakefulness
Delayed recall: No interaction between group and condition $(\mathrm{E} 5, \mathrm{R} 1, \mathrm{TSD}, \mathrm{R} 2)$ was found $\left(F(3,86.2)=1.06, p=.37, \omega^{2}=.001\right.$, $\left.\eta_{p}{ }^{2}=.023\right)$. The number of forgotten words in the morning did not differ between the control and chronic sleep restriction group ( $F(1$, $31.2)=0.59, p=.45$ ). Information on the Cls is given in Table S1. However, it differed depending on the condition $(F(3,86.2)=10.79$, $p<.0001)$. Within the control group mixed ANOVAs indicated an effect $(F(4,50)=3.9, p=.009)$ of condition (B2, E5, R1, TSD, R2). Post hoc Ismeans comparing differences to baseline indicated impaired performance during acute TSD compared to baseline ( $p=$ .01). The chronic sleep restriction group also showed an effect of condition $(F(4,66)=8.68, p<.0001)$. Post hoc tests indicated significantly more forgotten word pairs during acute sleep deprivation compared to baseline performance $(p<.0001)$. Figure 4 gives an overview of delayed recall performance per group. For information on performance across all experimental days (B2, E1-E5, R1, TSD, R2) see Figure S2.

\section{3 | Polysomnography}

Descriptive statistics of sleep parameters and respective MannWhitney $U$-tests of group differences, as well as Wilcoxon tests of between-group comparisons, are shown in Table 1. Differences in sleep parameters between the groups at baseline appeared only concerning the amount of N3. By chance, individuals with a high N3 amount were predominantly present in the chronic sleep restriction group. The difference in the amount of N3-sleep was not driven by single participants. On E5, all sleep parameters differed between groups.

Sleep architecture of E5 and of the recovery nights (R1 and R2) differed from the baseline night within the chronic sleep restriction group. On E5, there was a significant decrease in SPT, Wake, N1, N2, REM and SOL compared to baseline. Minutes spent in N3, as well as N3 latency, showed no significant difference between those nights (respectively: $Z=-.24, p=.82$; $Z=-2.14, p=.03)$. As expected, the respective night did not differ from baseline in the control group. On recovery night 1 , decreased N1 and SOL compared to baseline were observed in the chronic sleep restriction group. On recovery night 2 , the chronic sleep restriction group showed increased SPT, N2, N3 and REM compared to baseline; whereas N1, SOL and N3 latency were decreased and Wake showed no significant difference ( $Z=-1.76, p=.08$ ). During recovery night 2 the control group had shortened latencies and a decreased amount of N1, whereas the amounts of N2 and N3 were increased. The control group did not show the REM rebound that was present in the chronic sleep restriction group. The amount of minutes spent in N3 on the fifth sleep-restricted night did not correlate with either $N$-back performance (letter $N$-back: omissions: $r(21)=$ -.16, $p=.49$; sensitivity: $r(21)=.12, p=.59$; spatial $N$-back: omissions: $r(21)=-.23, p=.32$; sensitivity: $r(21)=.16, p=.5$ ) or word recall $(r(18)=-.17, p=.5)$. 
FIGURE 4 Comparison of word recall during baseline 2 and key conditions in the control group and in the chronic sleep restriction group. The difference between correctly recalled words in the evening and in the morning is displayed for control (white) and chronic sleep restriction (grey) group separately. The mean is indicated by " $x$ ". ${ }^{* *} p<.0001,{ }^{*} p<.05$, Ismeans statement $t$-test with Bonferroni-Holm correction; B, baseline 2; E5, experimental night 5; R1, 2, recovery 1, 2; TSD, total sleep deprivation

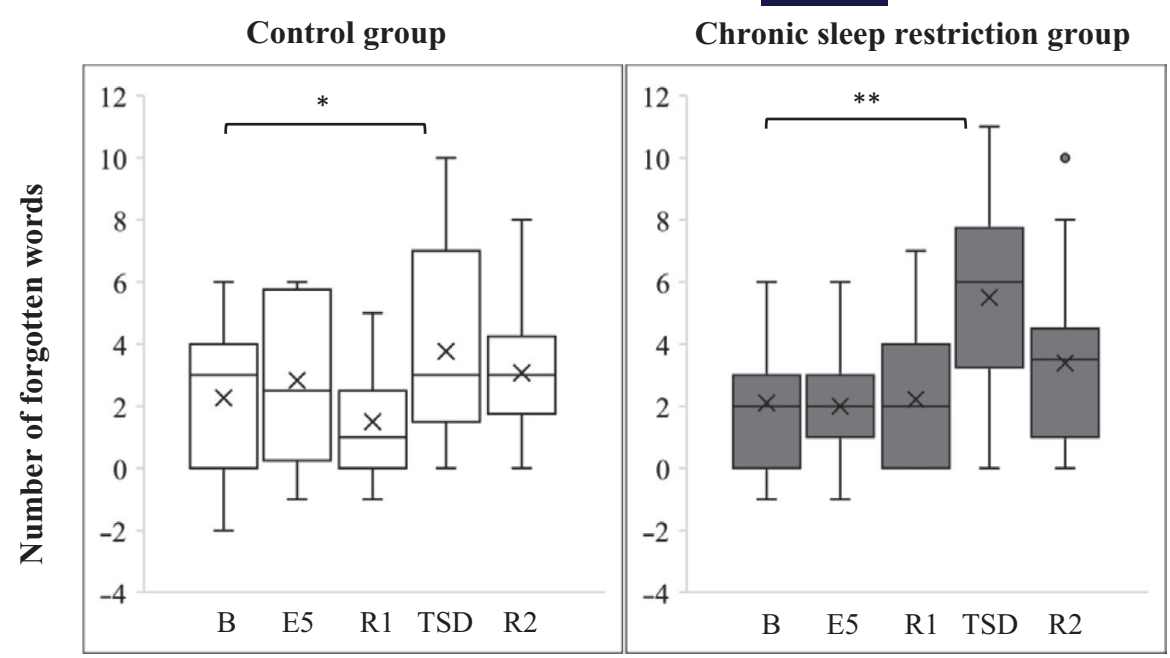

\begin{tabular}{|c|c|c|c|c|c|}
\hline \multirow[b]{2}{*}{ Sleep parameter } & \multirow[b]{2}{*}{ Group } & \multicolumn{4}{|c|}{ Condition, mean (SE) } \\
\hline & & Baseline & $\begin{array}{l}\text { Experimental } \\
\text { night } 5\end{array}$ & Recovery 1 & Recovery 2 \\
\hline \multirow[t]{2}{*}{$\mathrm{SPT}, \min$} & Control & $450.7(5.3)$ & $438.6(8.5) \Delta$ & $445.3(6.3) \Delta$ & $587.1(2.9) \Delta^{*}$ \\
\hline & CSR & $452.9(3.5)$ & $289.6(1.2)^{*}$ & $461(5.2)^{*}$ & $595(0.5)^{*}$ \\
\hline \multirow[t]{2}{*}{$\mathrm{N} 1, \min$} & Control & $22.1(1.8)$ & $20.2(1.7) \Delta$ & $21.6(2.5) \Delta$ & $17.4(1.8)^{*}$ \\
\hline & CSR & $16.9(1.5)$ & $4.4(0.4)^{*}$ & $12.4(1.2)^{*}$ & $12(1.4)^{*}$ \\
\hline \multirow[t]{2}{*}{$\mathrm{N} 2, \min$} & Control & $205.9(8.9)$ & $203.9(7.7) \Delta$ & $201.3(8.5)$ & $288.8(9.5)^{*}$ \\
\hline & CSR & $196.8(6.1)$ & $104(4.5)^{*}$ & $192.9(6.5)$ & $268.2(5.6)^{*}$ \\
\hline \multirow[t]{2}{*}{ N3, min } & Control & $87.8(7.9) \Delta$ & $84.7(7.2) \Delta$ & $90.7(7.2) \Delta$ & $136.3(10.7)^{*}$ \\
\hline & CSR & $114.3(6.9)$ & $115.0(5.1)$ & $123.5(7.3)$ & $166.2(6.8)^{*}$ \\
\hline \multirow[t]{2}{*}{ REM, min } & Control & $106.2(4.7)$ & $107.9(5.7) \Delta$ & $101.2(5.5)$ & $121.9(6.3)$ \\
\hline & CSR & $99.3(3.2)$ & $59.2(3.6)^{*}$ & $109.1(4.5)$ & $127.7(6.2)^{*}$ \\
\hline \multirow[t]{2}{*}{ Wake, min } & Control & $28.7(3.2)$ & $21.9(2.5) \Delta$ & $30.4(4.9)$ & $22.8(2.6)$ \\
\hline & CSR & $25.6(2.8)$ & $7.1(1.4)^{*}$ & $23(3.7)$ & 20.9 (3.5) \\
\hline \multirow[t]{2}{*}{$\mathrm{SOL}, \min$} & Control & $24.8(3.9)$ & $35.5(7.9) \Delta$ & $31.5(5.8) \Delta$ & $8.3(1.3)^{*}$ \\
\hline & CSR & $26.4(3.4)$ & $10.4(1.1)^{*}$ & $19(5.2)^{*}$ & $5.5(0.5)^{*}$ \\
\hline \multirow[t]{2}{*}{ N3 latency, min } & Control & $10.6(1)$ & $10.4(1) \Delta$ & $10.8(1.4)$ & $5(0.5)^{*}$ \\
\hline & CSR & $9.2(1)$ & $7.7(0.9)$ & $11.8(3.4)$ & $4.6(0.4)^{*}$ \\
\hline
\end{tabular}

TABLE 1 Comparison of sleep characteristics in the different study conditions
Group means (standard errors) of minutes of sleep period time (SPT), sleep stages in sleep period time and sleep onset latency (SOL) as well as N3 latency of the second baseline night, experimental night 5 and recovery nights 1 and 2 are shown. Please see Figure 1 for time in bed information for the respective nights. Values are displayed separately for the control (Control) and chronic sleep restriction (CSR) group. Mann-Whitney U-tests between groups were performed to assess whether there were differences in sleep parameters during the nights. Significant differences $(p<.05)$ are indicated by $\Delta$ after correcting according to Bonferroni-Holm. Significant differences to baseline are indicated by an asterisk.

\section{4 | DISCUSSION}

In the present study, we investigated single and combined effects of chronic sleep restriction ( 5 nights at $5 \mathrm{hr}$ ) and acute TSD (38 hr) with an intermittent 8-hr recovery night on working memory, declarative memory, and sleep. A chronic sleep restriction group underwent both chronic and acute TSD whereas a control group underwent acute TSD only.

Acute TSD without prior sleep restriction was associated with impairments in working memory and declarative memory in accordance with the literature (Elmenhorst et al., 2017; Gais et al., 2006; Lo et al., 2012). 
Chronic sleep restriction led to a deterioration of spatial working memory performance, but not verbal working memory. Right hemisphere structures involved in spatial working memory might thus be more affected by restricted sleep than left brain structures involved in verbal working memory. Our present findings give no indication that chronic or acute sleep loss effects on $\mathrm{N}$-back performance are influenced by executive load of the respective task, as load of the $\mathrm{N}$-back did not show an interaction with condition. Our present results are partly reminiscent of those of Lo et al. (2012). They assessed the effect of multiple nights of chronic sleep restriction and additional acute sleep deprivation on different cognitive processes, and found an effect on $\mathrm{N}$-back performance, although performance decrements in this working memory test were smaller than on sustained attention tasks. This finding is interesting as it emphasises that working memory performance is not simply dependent on sustained attention. However, the results of Lo et al. (2012) may fit partly the controlled attention hypothesis (Lim \& Dinges, 2010; Pilcher et al., 2007), which assumes that engaging tasks are less affected by sleep deficits than monotonous tasks, as the latter have higher demands on top-down control. In contrast and consistent with our expectation, declarative memory recall was not affected during chronic sleep restriction (Cedernaes et al., 2016; Kopasz et al., 2010; Lo et al., 2016; Tucker \& Fishbein, 2009). The finding of impaired spatial working memory, but preserved verbal working memory function and declarative memory recall favour previous findings that cognitive domains show differential vulnerabilities to sleep loss (Lo et al., 2012). These differences presumably reflect various involvements of underlying brain structures and dependence on diverse sleep components. Furthermore, characteristics of task application might have contributed to this finding, as the declarative memory task was performed without much time pressure, whereas the working memory task was a continuous performance test. However, the latter explanation does not account for the contrary findings regarding the two versions of the $\mathrm{N}$-back task.

The primary focus of the present work was on potential adverse interaction effects of chronic and acute sleep loss on memory performance. Acute sleep loss led to worse spatial $\mathrm{N}$-back performance in individuals with prior sleep restriction compared to individuals without a sleep deficit. This performance degradation in the chronic sleep restriction group in comparison to the control group was uncovered in the course of acute wakefulness, as significant group differences in performance appeared after $32 \mathrm{hr}$ of wakefulness. There were no significant group differences in the morning measures during acute TSD. However, a medium effect size for the group difference at TSD regarding the letter $\mathrm{N}$-back omissions was found. Neither verbal working memory nor declarative memory seemed to be affected by chronic sleep restriction, which was supported by low effect sizes.

Importantly, these interaction effects of acute and chronic sleep loss on spatial working memory appeared despite an intermittent night of recovery sleep between chronic and acute sleep deprivation. Interaction effects were for example shown by Lo et al. (2012), but there was no recovery between chronic and acute sleep loss. Whereas 1 night of recovery sleep after chronic sleep restriction appeared to temporarily restore spatial working memory performance in the subsequent morning in our present study, residual chronic sleep debt still rendered individuals more vulnerable to the effects of subsequent acute sleep loss. Cohen et al. (2010) exposed participants to chronic sleep loss and subsequent recovery sleep. After recovery sleep, vigilance performance was restored for several hours, but thereafter deteriorated at a faster rate. The authors suggest the existence of two different homeostatic processes to explain their findings: an acute homeostatic process acting on a short time scale and a chronic sleep homeostatic process acting on a longer time scale. Our present results complement their work supporting the existence of a second process accounting for chronic sleep deficits, which also seems to be implicated in working memory functions. With regard to demands in shift work, our present results implicate possible risks to work safety. Whereas performance early into a wake episode appears to reflect the immediate benefits of the passed sleep episode independent of the presence of chronic sleep debt, the latter exacerbates performance impairment as the wake episode progresses. Thus, performance after recovery sleep might initially give a false sense of recuperation (Cohen et al., 2010). This tendency is likely to be reinforced by subjective underestimations of sleepiness as compared to objective measures during chronically restricted sleep (Van Dongen et al., 2003).

Some limitations of our present study should be considered. Regarding the paired-associate list task, our study design did not exclude the possibility of impaired recall due to sleepiness. However, Gais et al. (2006) provided evidence that memory recall after acute sleep deprivation is not solely impaired due to sleepiness. The 11-day stay in the laboratory under controlled environmental conditions, a prescribed time schedule, away from friends and families constitute exceptional circumstances. The transfer of the reported results to memory performance in everyday life and thus the generalisability of the results should be treated with caution.

In conclusion, the present study suggests that 5 nights of sleep restriction negatively impact spatial working memory performance, but not verbal working memory or the delayed recall of declarative memories. Moreover, acute TSD led to larger impairments in spatial working memory in individuals with a history of sleep restriction than in individuals without sleep restriction. Prior sleep restriction did not affect verbal working memory performance and declarative memory recall during acute TSD.

Interaction effects of chronic and acute sleep deprivation on spatial working memory were evident in the present study despite an intermittent recovery night. These results clearly suggest that a sleep deficit accumulated across several nights of shortened sleep cannot be recovered completely within a single regular night of sleep. Our present results are of importance for shift work planning. Shift workers often suffer from chronically accumulated sleep deficits, as sleep opportunities take place at adverse circadian times. Furthermore, our observation of a temporary improvement in the morning on the day following a single recovery sleep 
episode implies that individuals might get a false sense of sufficient recuperation, while remaining more vulnerable to the impact of extended wakefulness. Adequate recovery periods should be provided to avoid cognitive impairments, especially when extended wake durations are a part of work schedules. These findings highlight the importance of extended recovery periods to prevent errors and accidents.

\section{ACKNOWLEDGEMENTS}

The authors gratefully thank their colleagues from the DLR division of Sleep and Human Factors Research and from Forschungszentrum Jülich (INM-2), especially for excellent support in the conductance of the study, data processing and EEG scoring. We would like to thank Jan Wagner and Werner Plihal from Lübeck University for providing us with word pairs for the declarative memory task. We thank Marie-Therese Puth (Institute for Medical Biostatistics, Medical Informatics and Medical Epidemiology of the Bonn University) for her statistical support.

\section{CONFLICT OF INTEREST}

No conflicts of interest declared.

\section{AUTHOR CONTRIBUTIONS}

$\mathrm{DE}, \mathrm{AB}, \mathrm{DA}$ and E-ME designed research; EH, DL, FS, JF, DE and $\mathrm{E}-\mathrm{ME}$ performed research; $\mathrm{EH}$ and $\mathrm{E}-\mathrm{ME}$ analysed data; $\mathrm{EH}$ wrote the paper; EH, DL, FS, JF, DE, AB, DA and E-ME revised the paper.

\section{DATA AVAILABILITY STATEMENT}

The data that support the findings of this study are available from the corresponding author upon reasonable request.

\section{ORCID}

Eva Hennecke (iD) https://orcid.org/0000-0002-7717-8937 Eva-Maria Elmenhorst (iD https://orcid.org/0000-0003-0336-6705

\section{REFERENCES}

Aeschbach, D., \& Borbely, A. A. (1993). All-night dynamics of the human sleep EEG. Journal of Sleep Research, 2, 70-81. https://doi. org/10.1111/j.1365-2869.1993.tb00065.x

Aeschbach, D., Cutler, A. J., \& Ronda, J. M. (2008). A role for non-rapid-eyemovement sleep homeostasis in perceptual learning. Journal of Neuroscience, 28, 2766-2772. https://doi.org/10.1523/jneurosci.5548-07.2008

Backhaus, J., Born, J., Hoeckesfeld, R., Fokuhl, S., Hohagen, F., \& Junghanns, K. (2007). Midlife decline in declarative memory consolidation is correlated with a decline in slow wave sleep. Learning \& Memory, 14, 336-341. https://doi.org/10.1101/Im.470507

Baddeley, A. (2010). Working memory. Current Biology, 20, R136-R140. https://doi.org/10.1016/j.cub.2009.12.014

Baddeley, A. D., \& Hitch, G. J. (1974). Working memory. In G. A. Bower (Ed.), Recent advances in learning and motivation (Vol. 8, pp. 47-89). Academic Press. https://doi.org/10.4324/9781315111261-7

Basner, M., Müller, U., \& Elmenhorst, E.-M. (2011). Single and combined effects of air, road, and rail traffic noise on sleep and recuperation. Sleep, 34, 11-23. https://doi.org/10.1093/sleep/34.1.11

Belenky, G., Wesensten, N. J., Thorne, D. R., Thomas, M. L., Sing, H. C., Redmond, D. P., Russo, M. B., \& Balkin, T. J. (2003).
Patterns of performance degradation and restoration during sleep restriction and subsequent recovery: A sleep dose-response study. Journal of Sleep Research, 12, 1-12. https://doi. org/10.1046/j.1365-2869.2003.00337.x

Borbely, A. A. (1982). A two process model of sleep regulation. Human Neurobiology, 1, 195-204.

Borbely, A. A., Baumann, F., Brandeis, D., Strauch, I., \& Lehmann, D. (1981). Sleep deprivation: Effect on sleep stages and EEG power density in man. Electroencephalography and Clinical Neurophysiology, 51, 483-495. https://doi.org/10.1016/0013-4694(81)90225-x

Cedernaes, J., Sand, F., Liethof, L., Lampola, L., Hassanzadeh, S., Axelsson, E. K., Yeganeh, A., Ros, O., Broman, J.-E., Schiöth, H. B., \& Benedict, C. (2016). Learning and sleep-dependent consolidation of spatial and procedural memories are unaltered in young men under a fixed short sleep schedule. Neurobiology of Learning and Memory, 131, 87-94. https://doi.org/10.1016/j.nlm.2016.03.012

Chee, M. W., \& Choo, W. C. (2004). Functional imaging of working memory after $24 \mathrm{hr}$ of total sleep deprivation. Journal of Neuroscience, 24, 4560-4567. https://doi.org/10.1523/jneurosci.0007-04.2004

Cohen, D. A., Wang, W., Wyatt, J. K., Kronauer, R. E., Dijk, D.-J., Czeisler, C. A., \& Klerman, E. B. (2010). Uncovering residual effects of chronic sleep loss on human performance. Science Translational Medicine, 2, 14ra3. https://doi.org/10.1126/scitranslmed.3000458

Diekelmann, S., \& Born, J. (2010). The memory function of sleep. Nature Reviews Neuroscience, 11, 114-126. https://doi.org/10.1038/nrn2762

Dinges, D. F., Pack, F., Williams, K., Gillen, K. A., Powell, J. W., Ott, G. E., Aptowicz, C., \& Pack, A. I. (1997). Cumulative sleepiness, mood disturbance, and psychomotor vigilance performance decrements during a week of sleep restricted to 4-5 hours per night. Sleep, 20, 267-277. https://doi.org/10.1093/sleep/20.4.267

Ellis, P. D. (2010). The essential guide to effect sizes: Statistical power, meta-analysis, and the interpretation of research results. Cambridge University Press.

Elmenhorst, D., Elmenhorst, E. M., Hennecke, E., Kroll, T., Matusch, A., Aeschbach, D., \& Bauer, A. (2017). Recovery sleep after extended wakefulness restores elevated A1 adenosine receptor availability in the human brain. Proceedings of the National Academy of Sciences of the United States of America, 114, 4243-4248. https://doi.org/10.1073/ pnas. 1614677114

Elmenhorst, E. M., Elmenhorst, D., Benderoth, S., Kroll, T., Bauer, A., \& Aeschbach, D. (2018). Cognitive impairments by alcohol and sleep deprivation indicate trait characteristics and a potential role for adenosine A1 receptors. Proceedings of the National Academy of Sciences of the United States of America, 115, 8009-8014. https://doi. org/10.1073/pnas.1803770115

Elmenhorst, E. M., Elmenhorst, D., Luks, N., Maass, H., Vejvoda, M., \& Samel, A. (2008). Partial sleep deprivation: Impact on the architecture and quality of sleep. Sleep Medicine, 9(8), 840-850. https://doi. org/10.1016/j.sleep.2007.07.021

Gais, S., \& Born, J. (2004). Declarative memory consolidation: Mechanisms acting during human sleep. Learning \& Memory, 11, 679-685. https:// doi.org/10.1101/Im.80504

Gais, S., Lucas, B., \& Born, J. (2006). Sleep after learning aids memory recall. Learning \& Memory, 13, 259-262. https://doi.org/10.1101/ Im.132106

Gevins, A., \& Cutillo, B. (1993). Spatiotemporal dynamics of component processes in human working memory. Electroencephalography and Clinical Neurophysiology, 87, 128-143. https://doi.org/10.1016/00134694(93)90119-g

Gohar, A., Adams, A., Gertner, E., Sackett-Lundeen, L., Heitz, R., Engle, R., Haus, E., \& Bijwadia, J. (2009). Working memory capacity is decreased in sleep-deprived internal medicine residents. Journal of Clinical Sleep Medicine, 5, 191-197. https://doi.org/10.5664/jcsm.27484

Groeger, J. A., Viola, A. U., Lo, J. C., von Schantz, M., Archer, S. N., \& Dijk, D. J. (2008). Early morning executive functioning during sleep 
deprivation is compromised by a PERIOD3 polymorphism. Sleep, 31, 1159-1167.

Hartshorne, J. K., \& Makovski, T. (2019). The effect of working memory maintenance on long-term memory. Memory \& Cognition, 47, 749763. https://doi.org/10.3758/s13421-019-00908-6

Hirshkowitz, M., Whiton, K., Albert, S. M., Alessi, C., Bruni, O., DonCarlos, L., Hazen, N., Herman, J., Adams Hillard, P. J., Katz, E. S., KheirandishGozal, L., Neubauer, D. N., O’Donnell, A. E., Ohayon, M., Peever, J., Rawding, R., Sachdeva, R. C., Setters, B., Vitiello, M. V., \& Ware, J. C. (2015). National Sleep Foundation's updated sleep duration recommendations: Final report. Sleep Health, 1, 233-243. https://doi. org/10.1016/j.sleh.2015.10.004

Iber, C., Ancoli-Israel, S., Chesson, A., \& Quan, S. F. (2007). The AASM manual for the scoring of sleep and associated events: Rules, terminology and technical specifications (1st ed.). American Academy of Sleep Medicine.

Jiang, F., VanDyke, R. D., Zhang, J., Li, F., Gozal, D., \& Shen, X. (2011). Effect of chronic sleep restriction on sleepiness and working memory in adolescents and young adults. Journal of Clinical and Experimental Neuropsychology, 33, 892-900. https://doi.org/10.1080/13803 395.2011.570252

Kirk, R. E. (1996). Practical significance: A concept whose time has come. Educational and Psychological Measurement, 56(5), 746-759. https:// doi.org/10.1177/0013164496056005002

Kopasz, M., Loessl, B., Valerius, G., Koenig, E., Matthaeas, N., Hornyak, M., Kloepfer, C., Nissen, C., Riemann, D., \& Voderholzer, U. (2010). No persisting effect of partial sleep curtailment on cognitive performance and declarative memory recall in adolescents. Journal of Sleep Research, 19, 71-79. https://doi.org/10.1111/j.1365-2869.2009.00742.x

Lim, J., \& Dinges, D. F. (2010). A meta-analysis of the impact of shortterm sleep deprivation on cognitive variables. Psychological Bulletin, 136, 375-389. https://doi.org/10.1037/a0018883

Lo, J. C., Bennion, K. A., \& Chee, M. W. (2016). Sleep restriction can attenuate prioritization benefits on declarative memory consolidation. Journal of Sleep Research, 25, 664-672. https://doi.org/10.1111/jsr.12424

Lo, J. C., Groeger, J. A., Santhi, N., Arbon, E. L., Lazar, A. S., Hasan, S., von Schantz, M., Archer, S. N., \& Dijk, D.-J. (2012). Effects of partial and acute total sleep deprivation on performance across cognitive domains, individuals and circadian phase. PLoS One, 7, e45987. https:// doi.org/10.1371/journal.pone.0045987

Pilcher, J. J., Band, D., Odle-Dusseau, H. N., \& Muth, E. R. (2007). Human performance under sustained operations and acute sleep deprivation conditions: Toward a model of controlled attention. Aviation, Space and Environmental Medicine, 78, 15-24.

Plihal, W., \& Born, J. (1997). Effects of early and late nocturnal sleep on declarative and procedural memory. Journal of Cognitive Neuroscience, 9, 534-547. https://doi.org/10.1162/jocn.1997.9.4.534

Rasch, B., \& Born, J. (2013). About sleep's role in memory. Physiological Reviews, 93, 681-766. https://doi.org/10.1152/physrev.00032.2012

Smith, E. E., Jonides, J., \& Koeppe, R. A. (1996). Dissociating verbal and spatial working memory using PET. Cerebral Cortex, 6, 11-20. https:// doi.org/10.1093/cercor/6.1.11
Stanislaw, H., \& Todorov, N. (1999). Calculation of signal detection theory measures. Behavior Research Methods, Instruments, \& Computers, 31, 137-149. https://doi.org/10.3758/bf03207704

Tippey, K. G., \& Longnecker, M. T. (2016). An ad hoc method for computing pseudo-effect size for mixed models. Retrieved from http:// www.scsug.org/wp-content/uploads/2016/11/Ad-Hoc-Metho d-for-Computing-Effect-Size-for-Mixed-Models_PROCEEDING S-UPDATE-1.pdf

Tucker, A. M., Whitney, P., Belenky, G., Hinson, J. M., \& Van Dongen, H. P. (2010). Effects of sleep deprivation on dissociated components of executive functioning. Sleep, 33, 47-57. https://doi.org/10.1093/ sleep/33.1.47

Tucker, M. A., \& Fishbein, W. (2009). The impact of sleep duration and subject intelligence on declarative and motor memory performance: How much is enough? Journal of Sleep Research, 18, 304-312. https:// doi.org/10.1111/j.1365-2869.2009.00740.x

Tucker, M. A., Hirota, Y., Wamsley, E. J., Lau, H., Chaklader, A., \& Fishbein, W. (2006). A daytime nap containing solely non-REM sleep enhances declarative but not procedural memory. Neurobiology of Learning and Memory, 86, 241-247. https://doi.org/10.1016/j.nlm.2006.03.005

Van Dongen, H. P., Maislin, G., Mullington, J. M., \& Dinges, D. F. (2003). The cumulative cost of additional wakefulness: Dose-response effects on neurobehavioral functions and sleep physiology from chronic sleep restriction and total sleep deprivation. Sleep, 26, 117126. https://doi.org/10.1093/sleep/26.2.117

Voderholzer, U., Piosczyk, H., Holz, J., Landmann, N., Feige, B., Loessl, B., Kopasz, M., Doerr, J. P., Riemann, D., \& Nissen, C. (2011). Sleep restriction over several days does not affect long-term recall of declarative and procedural memories in adolescents. Sleep Medicine, 12, 170-178. https://doi.org/10.1016/j.sleep.2010.07.017

Watson, N. F., Badr, M. S., Belenky, G., Bliwise, D. L., Buxton, O. M., Buysse, D., Dinges, D. F., Gangwisch, J., Grandner, M. A., Kushida, C., Malhotra, R. K., Martin, J. L., Patel, S. R., Quan, S., \& Tasali, E. (2015). Recommended amount of sleep for a healthy adult: A joint consensus statement of the american academy of sleep medicine and sleep research society. Sleep, 38, 843-844. https://doi.org/10.5665/ sleep.4716

\section{SUPPORTING INFORMATION}

Additional supporting information may be found online in the Supporting Information section.

How to cite this article: Hennecke E, Lange D, Steenbergen

$\mathrm{F}$, et al. Adverse interaction effects of chronic and acute sleep deficits on spatial working memory but not on verbal working memory or declarative memory. J Sleep Res.

2020;00:e13225. https://doi.org/10.1111/jsr.13225 